\title{
Orientation-sensed optomechanical accelerometers based on exceptional points
}

\author{
Rodion Kononchuk ๑* and Tsampikos Kottos $\odot$ \\ Wave Transport in Complex Systems Lab, Department of Physics, Wesleyan University, Middletown, Connecticut 06459, USA
}

(Received 1 February 2020; revised manuscript received 22 April 2020; accepted 4 May 2020; published 29 May 2020)

\begin{abstract}
We propose an optomechanical design, consisting of a parity-time symmetric multilayer structure tuned at exceptional-point degeneracy (EPD), with an adjustable layer that is coupled to micromechanical springs. The deflections of this layer in response to accelerations $\alpha$ lead to square-root resonance detuning $\Delta \omega \equiv \omega-\omega_{\mathrm{EPD}}=$ $\sqrt{\alpha}$ - thus dramatically enhancing the probe of ultrasmall accelerations $\alpha \ll 1$. Our design is scalable and can, in principle, support higher $N$ th-order EPDs with sensitivity $\Delta \omega \propto \sqrt[n]{\alpha}$. It also provides a pathway toward the next generation of on-chip hypersensitive accelerometers and vibrometers.
\end{abstract}

DOI: 10.1103/PhysRevResearch.2.023252

\section{INTRODUCTION}

The monitoring of directional acceleration is essential for a variety of technological applications, ranging from navigation devices, gravity gradiometry, and earthquake monitoring, to intruder detection, airbag deployment sensors in automobiles, and consumer electronics protection [1-4]. Depending on the application at hand, the requirements of the performance metrics of the various types of accelerometers vary. Nevertheless, most of the existing schemes utilize a linear-response principle to measure a constant acceleration. These measurement protocols involve a mounted test-mass whose displacement, due to the applied acceleration, is typically proportional to the acceleration, and it is sensed using capacitive [5], piezoelectric [6], tunnel current [7], or optical methods [8-13]. A bottleneck for all these schemes is the accuracy of the displacement sensor, which constitutes a central figure of merit of the accelerometers. Needless to say, optical sensing schemes have so far been proven superior (as far as resolution is concerned) to all others. In fact, recent developments in optomechanics have spurred the realization of the next generation of photonic motion detectors that surpass the sensitivity limitations of the current standards, with an aim to approach fundamental limits set by quantum mechanics $[14,15]$. These systems rely on the coupling between mechanical motion and light, which is enhanced when using optical resonators such as a Fabry-Pérot cavity [16], nanocavities in the form of photonic crystals [12,17], or by using quantum-correlated light [18,19]. Nevertheless, the requirement for accurate displacement measurements of the test-mass remains a daunting task.

In this paper, we propose a class of on-chip optomechanical accelerometers that utilize concepts from non-Hermitian

\footnotetext{
*rkononchuk@wesleyan.edu

Published by the American Physical Society under the terms of the Creative Commons Attribution 4.0 International license. Further distribution of this work must maintain attribution to the author(s) and the published article's title, journal citation, and DOI.
}

wave mechanics in order to achieve enhanced displacement measurements of the test-mass. Specifically, we exploit the rich mathematical structures underlying the presence of nonHermitian spectral degeneracies, known as exceptional points (EPs). These are branch point singularities in the parameter space of a system at which $N$ eigenvalues and their associate eigenvectors coalesce and become degenerate. This class of degeneracies has spawned significant interest and developments in optics and photonics [20,21], electronics [22], and acoustics [23]. As opposed to Hermitian degeneracies, where the modes are expanded around their degenerate point in a Taylor series with respect to a small perturbation $\epsilon$, here the appropriate expansion is the Puiseux series. In particular, for a system supporting an $N$ th-order EP, the frequency shift in response to a small perturbation $\omega_{n}=\omega_{\mathrm{EP}}+\sum_{m=1}^{\infty} c_{m}^{(n)} \epsilon^{m / N}$. Given that $\epsilon^{1 / N} \gg \epsilon$ for $|\epsilon| \ll 1$, such a non-Hermitian response opens up new horizons in probing small perturbations [24-30]. In fact, this concept has already been implemented for the development of a new generation of hypersensitive ring laser gyroscopes [31,32].

Here we propose an optomechanical EP-accelerometer consisting of a multilayer photonic crystal (PC) grown on a silicon platform [33,34]; see Fig. 1. The photonic crystal consists of quarter-wavelength silicon layers separated by air spacers. At symmetric positions with respect to the mirror plane of the structure, we placed two optically identical silicon defect layers, each having half-wavelength width. One of these layers $(S 1)$ has loss, whose origin can be traced either to radiation losses or to absorption, while the other one $(S 2)$ has an equal amount of gain. The gain can be introduced to the layer $S 2$ by injection of erbium $\mathrm{Er}^{3+}$ nanoparticles [35-37], which are subsequently pumped optically from the top. To prevent exposure of the rest of the layers from the pump, one has to apply to them a polymer mask from the top. Alternatively, the multilayer structure can be pumped from the right port at a band-gap wavelength, which is different from the defect resonant wavelength. In this case, the intensity of the pumped beam will decay exponentially inside the multilayer structure (since its wavelength is within the band gap), thus allowing us to pump the two cavities in a dramatically different 


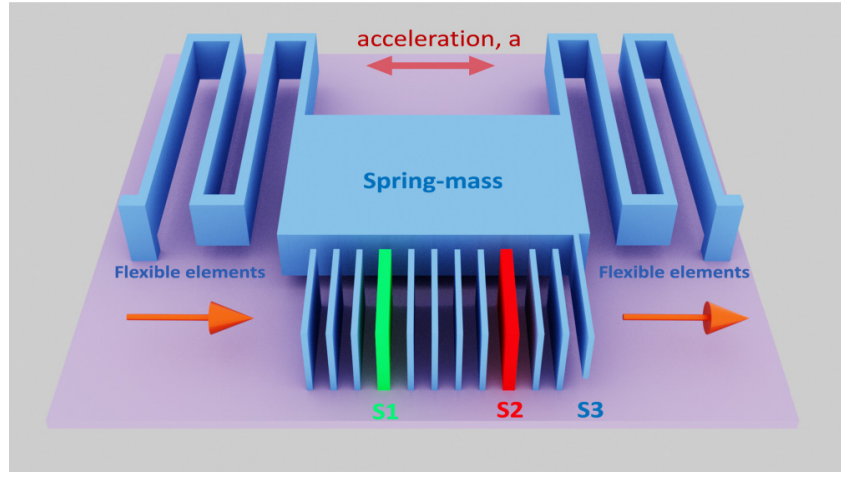

FIG. 1. (a) Schematic of the proposed design of an optomechanical accelerometer, consisting of a pair of Fabry-Pérot multilayer cavities with one of the cavities ( $S 1$ ) having losses $\gamma$ (green) and the second (S2) having an equal amount of gain (red). The cavities are grown on a silicon platform forming a silicon/air multilayer. One of the silicon layers (S3) is attached to the spring-mass (blue) in order to sense the in-plane acceleration by perturbing the resonant frequency of one of the modes. The spring-mass system senses an in-plane directional acceleration. It consists of flexible silicon elements and a test mass connected to the silicon layer (S3). The acceleration is probed by transmittance of the spectral measurements through the multilayer: left and right arrows denote the incident and transmitted light, correspondingly.

manner. Specifically, such a pumping protocol will provide exponentially small gain to the layer $S 1$. The total structure demonstrates a parity-time $(\mathcal{P} T)$ symmetry when the gain in layer $S 2$ balances the amount of losses experienced by the $S 1$ layer. The condition associated with balanced gain and loss cavities can in principle be realized by monitoring the transmittance spectra of the system while increasing the power of the pumped beam. In general, this PC supports two defect modes at distinct frequencies. When, however, the amount of gain/loss at each defect cavity matches the tunneling coupling between the (isolated) cavity modes, the system demonstrates an EP degeneracy. The tunneling coupling $\kappa$ between the modes residing at each of the two cavities is dictated by the number of bilayers between the defect layers $S 1$ and $S 2$. In particular, $\kappa \sim \frac{1}{\xi} e^{-N / \xi}$, where $N$ is the number of in-between layers and $\xi$ is the so-called localization length of the defect modes. At the same time, the losses at each cavity can be either Ohmic or radiative. The amount of radiative losses can be controlled, for example, by etching $S 1$ layer surfaces and thus introducing controllable imperfections, responsible for radiative losses.

Our design assumes that in the absence of acceleration, the system is at the EP. We have completed our design by further assuming that one of the silicon layers $(S 3)$ is attached to a spring mass, thus acting as a test-mass that senses in-plane accelerations; see Fig. 1. An in-plane acceleration in a direction parallel to the PC will cause the adjustable silicon layer to shift closer to (or farther from) the rest of the stack, depending on the direction of the acceleration $\alpha$. Alternatively, one may also consider a design in which the layer $S 3$ is stationary (grown on the substrate) while the rest of the stack is attached to a test-mass and senses the acceleration. In both cases, the applied acceleration enforces a thickness variation of the air spacer layer between the silicon layer $S 3$ and the rest of the stack. Consequently, this variation leads to a frequency detuning of the cavity formed by the defect layer $S 2$, and to a subsequent lift of the EP degeneracy. In particular, one of the emerging modes becomes high- $Q$ and experiences a resonant shift $\Delta \omega \propto \sqrt{\alpha}$ while the other one remains at $\omega_{\mathrm{EP}}$ and undergoes an abrupt overdamped transition. The proposed scheme is scalable, and the PC can be arranged to support an $N$-th order EP with higher-order sensitivity $\Delta \omega \propto \alpha^{1 / N}$ by introducing $N \mathcal{P} T$-symmetric defects. Additionally, the sensitivity of the device can be increased by modifying the design in such a way that few neighboring silicon layers are attached to the test-mass and thus move together farther from or closer to the rest of the stack once acceleration is applied. This will result in a thickness variation of the air spacer layer located closer to the defect silicon layer $S 2$. Since the electric field is exponentially enhanced in the vicinity of the defect layers, in this case the same thickness variation (applied acceleration) will lead to much stronger resonant shift detuning of the cavity formed by the layer $S 2$ compared with the case discussed above.

\section{DESIGN OF AN OPTOMECHANICAL PC}

We consider a PC consisting of quarter-wave silicon $(S)$ and air $(A)$ layers with the arrangement $(S A)^{3} S 1(A S)^{4} A S 2(A S)^{2} A 1 S 3$. The corresponding refractive indexes at an operational wavelength $\lambda \approx 1550 \mathrm{~nm}$ are $n_{S}=3.48$ and $n_{A}=1$. The defect layers $S 1$ and $S 2$ are half-wave-thick silicon layers with a complex index of refraction $n_{S 1}=3.48+0.00217765 i$ (losses) and $n_{S 2}=3.48-0.00217765 i \quad$ (gain), respectively [38]. In the numerics we modeled the (radiative or Ohmic) losses via an imaginary part of the refractive index. S3 is an adjustable quarter-wavelength silicon layer that is attached to a test-mass (Fig. 1) and it is underetched in order to avoid friction with the silicon surface-thus sensing the applied in-plane acceleration. Variations in its position due to in-plane acceleration lead to changes in the thickness of the quarter-wavelength air spacer $A 1$, and consequently to a resonant detuning of the $S 2$ defect layer. In general, the thickness of the silicon layers $S$ and $S 3$ should satisfy $n_{S} \times d_{S}=\frac{(2 \times N+1) \times \lambda_{0}}{4}, N \in \mathbb{Z}$, in order to support a Fabry-Pérot mode at operational wavelength $\lambda_{0}$, while the defect layers $S 1$ and $S 2$ should satisfy $n_{S 1,2} \times d_{S 1,2}=\frac{(2 \times N) \times \lambda_{0}}{4}, \quad N \in \mathbb{Z} \quad[33,34]$. This allows us to make the layers as thick as is required for a high-quality production process.

The micromechanical oscillator undergoing thermal and viscous damping due to the surrounding air has been designed using coupled structural mechanics and acoustic modules of COMSOL MULTIPHYSICS software [39]. It consists of the testmass made of silicon (blue) connected with an adjustable silicon layer $S 3$. The mass is coupled to a pair of flexible silicon elements (see Fig. 1). The size of the silicon mass is $500 \mu \mathrm{m} \times 250 \mu \mathrm{m} \times 5 \mu \mathrm{m}$, while the flexible elements consist of four silicon springs connected in series (as shown in Fig. 1) with dimensions $1 \mu \mathrm{m} \times 400 \mu \mathrm{m} \times 5 \mu \mathrm{m}$, separated by $15 \mu \mathrm{m}$ air gaps, responsible for thermal and viscous damping. For comparison, we also consider a design with the 

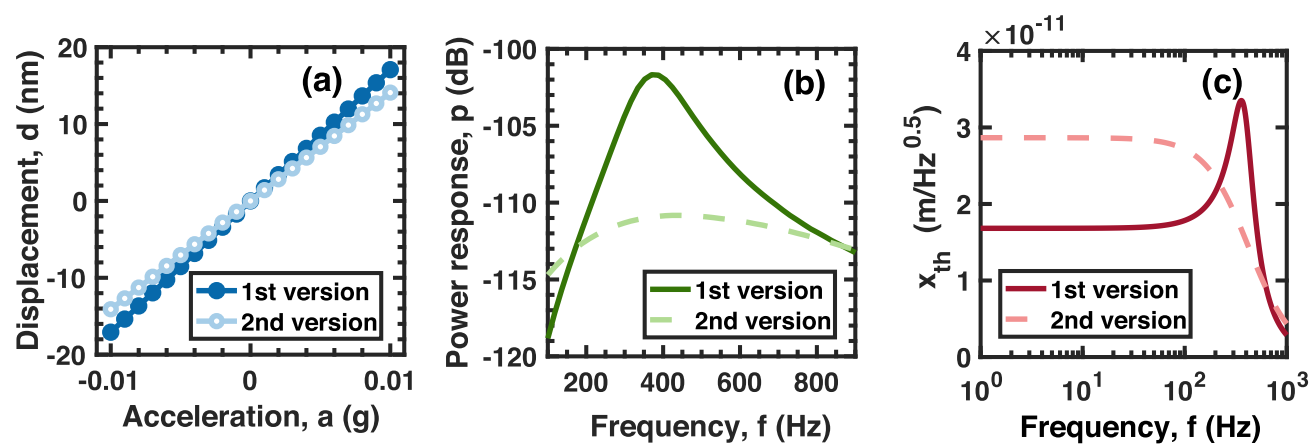

FIG. 2. (a) Simulated displacement $d$ of the layer $S 3$ attached to the spring mass, as a function of applied acceleration $\alpha$ for both versions of the spring mass (see text). The inverse slope in this diagram provides the value of the (angular) natural frequency of the mechanical oscillator $\omega=2.4 \mathrm{rad} \mathrm{kHz}$ for the first version of the design and $2.7 \mathrm{rad} \mathrm{kHz}$ for the second one. (b) The mechanical response of the oscillator of Fig. 1 . The mechanical linewidth is extracted from the resonant FWHM. (c) The spectral density of the thermal noise displacement for both versions of the mechanical oscillator of the proposed accelerometer.

following silicon spring parameters: $1 \mu \mathrm{m} \times 500 \mu \mathrm{m} \times 5 \mu \mathrm{m}$ and $11.6 \mu \mathrm{m}$ air gaps. The main requirements of the design were to guarantee (i) a uniform displacement of the $S 3$ layer for the range of accelerations that we have used, (ii) the validity of a linear response between the test-mass displacement and the acceleration in a low-frequency limit (see the Appendix A), i.e., $\alpha=\omega_{n}^{2} x_{\text {res }}$ (where $\omega_{n}$ is the angular natural frequency of the mechanical oscillator), and (iii) a low level of thermal noise. The displacement of the mass elements and of the silicon layer $S 3$, as a function of an applied horizontal inplane acceleration for both versions of the design, is shown in Fig. 2(a) and nicely demonstrates a linear response behavior, which allows us to extract the mechanical resonant frequency $\omega_{n}$, i.e., $\omega_{n} \equiv \sqrt{\frac{k_{\text {eff }}}{m_{\text {eff }}}}$, where $m_{\text {eff }} \approx 2.085 \mu \mathrm{g}$ is the effective mass and $k_{\mathrm{eff}} \approx 12.3 \mathrm{mN} \mathrm{m}^{-1}$ is the effective spring constant, while for the second version of the oscillator we obtain $m_{\mathrm{eff}} \approx$ $2.093 \mu \mathrm{g}$ and $k_{\text {eff }} \approx 14.9 \mathrm{mN} \mathrm{m}^{-1}$. These yield resonance frequencies of 386 and $424 \mathrm{~Hz}$, respectively. It is important to point out that small resonance frequencies $\omega_{n}$ are frequently incompatible with the necessity for high-speed operations. At the same time, large resonance frequencies $\omega_{n}$ lead to vanishingly small susceptibilities and therefore displacements. It is therefore imperative to develop measurements protocols that sense vanishingly small displacements.

Another important characteristic of the micromechanical oscillator is its mechanical $Q$-factor. The latter dictates the response time $\tau_{R}=Q / \omega_{n}$ of the oscillator to an external force [40]. In Fig. 2(b) we present a frequency domain simulation for the oscillator showing a power response $p=$ $20 \log _{10}(|\omega d|)$, which confirms the value of $\omega_{n}$ and allows us to extract the value of $Q \equiv \omega_{n} / \Delta \omega_{\mathrm{FWHM}}=1.92$ for the first version of the mechanical oscillator and $Q=0.5$ for the second one, corresponding to critical damping. In the latter case, the test-mass reaches the new equilibrium state without any oscillatory dynamics. Here $\Delta \omega_{\mathrm{FWHM}}$ is the full width at half-maximum (FWHM) and therefore $\tau_{R}=7.92 \times 10^{-4}$ and $1.95 \times 10^{-4} \mathrm{~s}$ of the first and second designs of the oscillator, respectively. The knowledge of these parameters provides an estimate of the lower bound sensitivity and noise effects on acceleration measurements. In particular, the fundamental resolution limit in acceleration measurements is typically set by the presence of thermal Brownian motion of the test-mass. The latter is quantified by the thermal noise equivalent acceleration $\alpha_{\mathrm{th}}$, which provides the lower bound in acceleration measurements $[41,42]$. It is given by (see the Appendix B)

$$
\alpha_{\mathrm{th}}=\sqrt{\frac{4 k_{b} T \omega_{n}}{m Q}}\left[\frac{g}{\sqrt{H z}}\right],
$$

where $k_{b}$ is the Boltzmann constant and $T=293.15 \mathrm{~K}$ is the temperature. In fact, there are two other noise sources related with (i) a readout noise $\left(\alpha_{\mathrm{det}}\right)$ and (ii) other noise sources $\left(\alpha_{\text {add }}\right)$ such as the ones associated with the use of the interrogating laser and the electronics used for the measurement process. However, these two types of noise sources can be minimized, leaving $\alpha_{\mathrm{th}}$ the main source that is responsible for bounding the resolution in acceleration measurements $[12,13]$. In fact, from Eq. (1) we see that a high resolution at room temperatures requires high values for the product $m Q$, i.e., both large mass and high mechanical $Q$-factor. Using the extracted value of $Q$, we find from Eq. (1) that $\alpha_{\mathrm{th}} \approx 10 \mu \mathrm{g} / \sqrt{\mathrm{Hz}}$ for the first version of the spring mass and $20 \mu \mathrm{g} / \sqrt{\mathrm{Hz}}$ for the second one (critically dumped mass), where $1 \mathrm{~g}$ stands for acceleration due to gravity. We conclude this analysis by pointing out that in our calculations for the $Q$ factor we took into consideration only the thermal and viscous damping due to the surrounding air. Contributions like the noncontact friction (in vacuum) associated with interactions between the adjustable layer $S 3$ and the silicon surface (or the silicon layer adjacent to $S 3$ ) are typically smaller than the air damping, and therefore they have been disregarded in our analysis. Using the above analysis, we can further evaluate the spectral density of the thermal noise displacement of the proposed accelerometer. It is given by (see the Appendix B)

$$
x_{\mathrm{th}}(\omega)=\sqrt{\frac{4 k_{b} T \omega_{n}}{m Q\left(\left(\omega_{n}^{2}-\omega^{2}\right)^{2}+\left(\frac{\omega_{n} \omega}{Q}\right)^{2}\right)}},
$$

and it is plotted in Fig. 2(c) for both design configurations. It shows almost flat spectral noise density at frequencies $f \leqslant 100 \mathrm{~Hz}$, implying that the maximum bandwidth of the proposed accelerometer is about $100 \mathrm{~Hz}$. The associated RMS noise acceleration of the first version of the mechanical 
oscillator, measured within this bandwidth $\Delta f=100 \mathrm{~Hz}$ (low pass filter), will be $\left\langle\alpha_{\text {noise }}\right\rangle=\alpha_{\mathrm{th}} \sqrt{\Delta f}=100 \mu \mathrm{g}$ (see the Appendix B), which sets the noise floor level [see the inset in Fig. 4(c)]. In contrast, the second (critically damped) oscillator design provides RMS noise acceleration that is twice as high, and thermal noise displacement that is about 1.7 times higher. At the same time, its higher resonant frequency leads to smaller sensitivity. When combined with small $Q$, this high resonant frequency leads to a faster response. In contrast, a higher $Q$ factor provides smaller thermal noise equivalent acceleration, which, of course, comes at the cost of the response time of the accelerometer. In the specific scenarios described here, the response time of both accelerometer configurations is significantly smaller than the inverse upper bound frequency discussed earlier in this paragraph $\left(f_{\max }=100 \mathrm{~Hz}\right)$, i.e., $\tau_{r} \ll 1 / f_{\max }$, and thus does not affect the measurements of accelerations with frequencies below this limit. This implies that the first (underdamped) version of the mechanical spring with $Q=1.92$ performs better as an accelerometer and will be the one considered further in the analysis below.

\section{PC WITH EXCEPTIONAL POINT DEGENERACY}

First we will investigate the existence of EP degeneracy for the $\mathcal{P} T$-symmetric PC. To this end, we analyze its transmission spectrum $T(\omega)$ in the absence of acceleration using transfer matrices. The latter connects the amplitudes of forward- and backward-propagating waves between two consequent layers (and between the first/last layer and the left/right ambient space). In particular, a time-harmonic field of frequency $\omega$ satisfies the following Helmholtz equation inside each spatial domain $0 \leqslant x_{j} \leqslant x_{j}+L_{j}\left(L_{j}\right.$ is the width of the $j$ th layer):

$$
\frac{d^{2} E(x)}{d x^{2}}+\left(\frac{\omega}{c}\right)^{2} \varepsilon(x) E(x)=0 .
$$

At the $j$ th layer, Eq. (3) admits solutions of the form $E^{(j)}(x)=$ $E_{f}^{(j)} e^{i n_{j} k x}+E_{b}^{(j)} e^{-i n_{j} k x}$, where $k=\omega / c$ is the free-space (air) wave vector. Similarly, on the left and right of the PC, Eq. (3) admits the solution $E^{(L / R)}(x)=E_{f}^{(L / R)} e^{i k x}+E_{b}^{(L / R)} e^{-i k x}$. The continuity of the field and its derivative at the interface between two layers (or a layer and the left-right ambient space) is expressed in terms of the total transfer matrix $M$, which connects the forward and backward amplitudes on the left $(L)$ and right $(R)$ of the $\mathrm{PC}$ :

$$
\left(\begin{array}{l}
E_{f}^{(R)} \\
E_{b}^{(R)}
\end{array}\right)=\left(\begin{array}{ll}
M_{11} & M_{12} \\
M_{21} & M_{22}
\end{array}\right)\left(\begin{array}{l}
E_{f}^{(L)} \\
E_{b}^{(L)}
\end{array}\right), M=\prod_{j=0}^{q} M_{j},
$$

where $q$ is the total number of layers. The single-layer transfer matrix $M_{j}$ connects the field amplitudes of the $j$ th and the $(j+1)$ th layers, i.e., $\left(E_{f}^{(j+1)}, E_{b}^{(j+1)}\right)^{T}=M_{j}\left(E_{f}^{(j)}, E_{b}^{(j)}\right)^{T}$. Thus, the transfer-matrix approach allows us also to construct the field $E^{(j)}(z)$ at each layer, provided that appropriate scattering boundary conditions are imposed. The latter, for a left incident wave, take the form $\left(E_{f}^{(R)}, E_{b}^{(R)}\right)^{T}=(1,0)^{T}$. It is easy to show that $T=\left|\frac{1}{M_{22}}\right|^{2}$, and $R=\left|\frac{M_{21}}{M_{22}}\right|^{2}[43,44]$.

We first analyze the transmission spectrum $T(\omega)$ in the case in which the two defect cavities $S 1$ and $S 2$ are identical, i.e., $\operatorname{Im}\left(n_{S 1}\right)=\operatorname{Im}\left(n_{S 2}\right)=0$. In this case, the cavities support pairs of identical cavity modes that are degenerate at $\omega_{0}$. When the two cavities are in proximity to one another, the cavity modes supported by each individual cavity are coupled together forming two "supermodes" with eigenfrequencies $\omega_{+}$and $\omega_{-}$[see Figs. 3(a), 3(b) and 3(c)]. In particular, the frequency splitting is controlled by the number of layers between the two defect cavities. In Fig. 3(a) we show the transmission spectrum of the PC with two identical defect layers. It shows two resonant peaks (associated with each of the two defect supermodes) forming close to the center of the band gap. The associated supermodes $\psi_{+}$and $\psi_{-}$are a linear (symmetric and antisymmetric) superposition of the individual cavity modes [see Figs. 3(b) and 3(c)]. When we introduce gain/loss to the two defect layers, i.e., $\operatorname{Im}\left(n_{S 1}\right)=$ $-\operatorname{Im}\left(n_{S 2}\right) \neq 0$, the two resonant peaks of $T(\omega)$ start approaching one another [see Fig. 3(d)]. This is a consequence of the $\mathcal{P} \mathcal{T}$-symmetry, which essentially renormalizes the coupling between the cavity modes, thus promoting the formation of an EP degeneracy. At the same time, the supermodes remain also eigenmodes of the $\mathcal{P} \mathcal{T}$-symmetric operator (albeit they are now complexed valued); see Figs. 3(e) and 3(f). When the gain/loss strength becomes equal to the coupling strength between the individual cavities, the two resonant peaks coalesce and form an EP degenerate pair at $\omega=\omega_{\mathrm{EP}}=\omega_{0}$; see Fig. 3(g). At the same time, the supermodes of the system become degenerate [Figs. 3(h) and 3(i)].

\section{EP-BASED OPTOMECHANICAL ACCELEROMETERS}

Next, we investigate the consequences of the acceleration $\alpha$ at the EP degeneracy. We note that for nonzero acceleration $\alpha \neq 0$, Eq. (3) has to be solved taking into account the adjusted equilibrium position of the $S 3$ layer [see Fig. 2(a)] and, consequently, the modified width $L_{A 1}$ of the last air spacer (A1). The latter can increase or decrease (with respect to its value for $\alpha=0$ ), depending on the orientation of the acceleration. Figures 4(a) and 4(b) show the transmittance $T(\lambda)$ for various values of the applied acceleration $\alpha$. We observe a (red/blue) shift of the resonant peak of $T(\lambda)$ depending on the direction of the acceleration. In Fig. 4(c) we present a panorama of these resonant shifts versus the acceleration. A fractional power-law behavior of the peak wavelength $\lambda_{\max }$ versus $\alpha$ is evident, i.e., $\lambda_{\max } \approx 2 \sqrt{\alpha}(\mathrm{g})$. For comparison purposes, we also plot in the same figure a standard linear dependence $\lambda_{\max } \approx 2 \alpha(\mathrm{g})$ occurring in the case of typical optomechanical accelerometers based on linear response. This comparison implies that the proposed EP optomechanical accelerometer has a dramatic sensitivity improvement as far as the displacement of the test mass is concerned. The inset in Fig. 4(c) illustrates the floor noise level (orange region) of the measured accelerations calculated from Eq. (1).

The theoretical analysis of the square-root response can be performed using a coupled mode theory (CMT). Since the cavity defect modes are located in the band gap and are well-separated from the rest of the Fabry-Pérot modes, we can eliminate them from our consideration. In the proposed multilayered circuit there are two distinct defect cavity modes, as shown on Fig. 3, formed by the defect layers $S 1$ and $S 2$. The $S 3$ layer is similar to the rest of the multilayer 

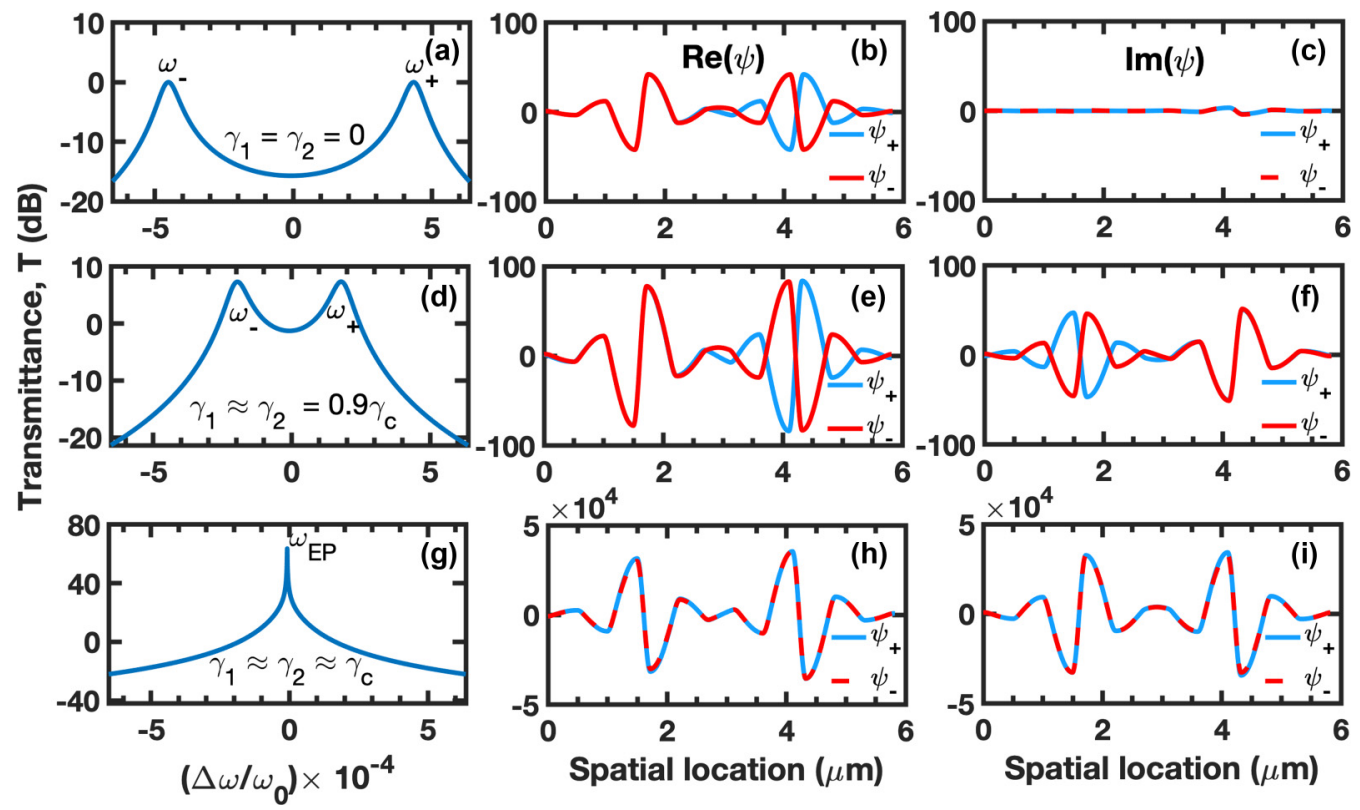

FIG. 3. (a) Simulated transmittance spectra of the multilayer, without gain and loss $\left(\gamma_{1}=\gamma_{2}=0\right)$ in the vicinity of the resonant frequency $\omega_{0}$. Corresponding spatial profile within the multilayer of the real (b) and imaginary (c) parts of forward-propagated wave functions $\psi_{+}$ and $\psi_{-}$. (d) Transmittance spectra of the multilayer, close to the EP condition $\left(\gamma_{1} \approx \gamma_{2}=0.9 \gamma_{c}\right)$. Corresponding spatial profile within the multilayer of the real (e) and imaginary (f) parts of forward-propagated wave functions $\psi_{+}$and $\psi_{-}$. (g) Transmittance spectra of the multilayer, very close to the EP condition $\left(\gamma_{1} \approx \gamma_{2} \approx \gamma_{c}\right)$. Corresponding spatial profile within the multilayer of the real (h) and imaginary (i) parts of forward-propagated wave functions $\psi_{+}$and $\psi_{-}$.

quarter-wave-thick $S$-layers, and its displacement is considered small enough. Therefore, it does not support any additional defect modes. It is only used as a test-mass in order to sense the acceleration. The two interacting (via tunneling) defect cavity modes are described by the following $2 \times 2$
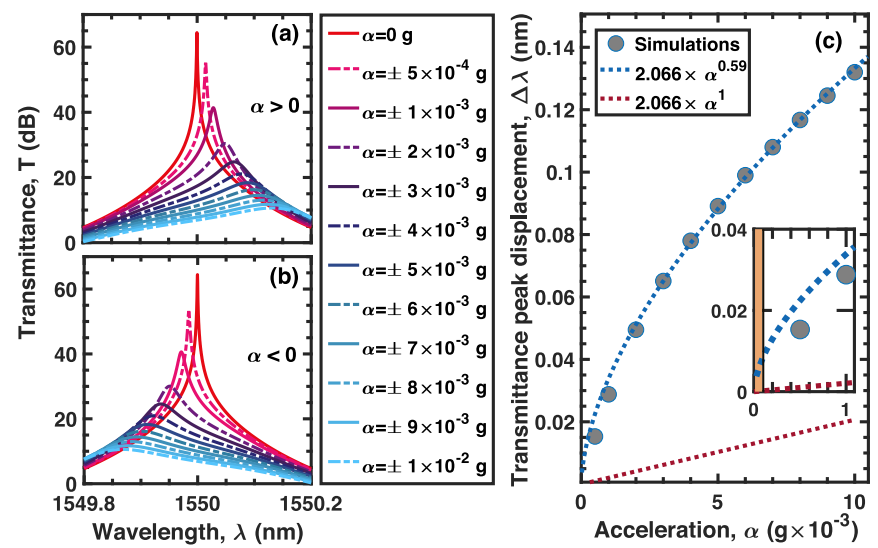

FIG. 4. (a) Simulated transmittance spectra of the optomechanical accelerometer schematically drawn in Fig. 1 with integrated spring-mass system for various small values of applied right-directed acceleration $(\alpha>0)$. (b) Simulated transmittance spectra for various small values of left-directed acceleration $(\alpha<0)$. (c) Transmittance peak displacement $\Delta \lambda$ from the EP spectral degeneracy obtained from panel (a) as a function of applied acceleration $\alpha$ (gray circular dots) and its best fit with the function $2.066 \times \alpha^{0.59}$ (dashed blue line). Linear function $2.066 \times \alpha$ (dashed red line) is added for comparison. The orange region in the inset indicates the RMS noise acceleration, (i.e., floor noise level). effective Hamiltonian:

$$
H_{0}=\left(\begin{array}{cc}
\omega_{0}-i \gamma_{1} & \kappa \\
\kappa & \omega_{0}+i \gamma_{2}
\end{array}\right),
$$

where $\omega_{0}$ is an eigenfrequency of the individual stand-alone cavity mode, $\gamma_{1,2}$ is their linewidth due to the presence of the loss/gain mechanisms at the first and second cavities, respectively, which in the case of a $\mathcal{P} T$ symmetric system with balanced gain and loss yields $\gamma=\gamma_{1}=\gamma_{2}$, and $\kappa$ is the coupling between the two cavities due to tunneling across the in-between layers of the PC. One can diagonalize Eq. (5) and evaluate the eigenfrequencies of the coupled cavity system:

$$
\omega_{ \pm}=\omega_{0} \pm \sqrt{\kappa^{2}-\gamma^{2}}
$$

with corresponding eigenvectors $v_{ \pm}=\left(\frac{-i \gamma \pm \sqrt{\kappa^{2}-\gamma^{2}}}{\kappa}, 1\right)^{T}$. The above equations imply that the two modes become degenerate, $\omega_{ \pm}=\omega_{\mathrm{EP}}=\omega_{0}$ (EP degeneracy), when $\kappa_{\mathrm{EP}} \equiv \kappa=\gamma$. We consider that the two cavity modes of the PC are satisfying the EP condition.

In the case of Fabry-Pérot multilayer cavities, a small perturbation of the thickness of the air spacer layer due to a displacement $x(\alpha) \ll 1$ of the $S 3$-layer leads to a linear response in the eigenfrequency of the supermode of the system, i.e., $\omega_{0} \rightarrow \omega_{0}+\epsilon$, where $\epsilon(\alpha)=K x(\alpha)$ [45]. The coefficient $K$ depends on the multilayer design and the exact location of the layer whose thickness is perturbed. At the same time, $x(\alpha)=\chi \alpha$ (see the Appendix A). Therefore, the eigenfrequency perturbation is a linear function of the applied acceleration, i.e., $\epsilon(\alpha)=K \chi \alpha$. This implies that the main source of noise in the acceleration measurements is related to the mechanical noise associated with the displacement of 
$S 3$. The latter subsequently affects the eigenfrequency perturbation $\epsilon$, which is connected with the noise equivalent acceleration $\alpha_{\text {noise }}$ as $\epsilon_{\text {noise }}=K \chi \alpha_{\text {noise }}$. Importantly, as is discussed in Sec. II, the spring-mass is designed to sense accelerations with frequencies below $f_{\max }=100 \mathrm{~Hz}$. This upper limit is 12 orders of magnitude lower than the optical eigenfrequencies of the multilayer $(199.9 \mathrm{THz})$. It implies that the mechanical oscillations of the $S 3$ layer due to acceleration are adiabatic with respect to the optical setup and thus do not affect either the $\mathcal{P} T$-symmetry of the system or the formation of the EP degeneracy.

Strictly speaking, a thickness variation of any single layer of a multilayer structure affects both supermodes of the system. The size of the perturbation with which a particular eigenfrequency is affected decays exponentially with the distance of the cavity (which supports this supermode) from the modulated layer. In the proposed design, the distance between the layer $S 3$ and the defect layer $S 1$ (supporting an eigenmode $H_{11}$ ) is three times larger than the distance between layers $S 3$ and $S 2$ (supporting an eigenmode $H_{22}$ ). In such a case, the acceleration will modify the elements of the effective Hamiltonian by $H_{11} \rightarrow \omega_{0}+\epsilon_{1}$ and $H_{22} \rightarrow \omega_{0}+\epsilon_{1}+\epsilon$, i.e., leading to a relative frequency shift $\epsilon$ between the two resonant modes. In fact, based on the previous discussion we can safely assume that $\epsilon_{1} \ll \epsilon \ll \omega_{0}$ and thus we can safely ignore the global shift $\epsilon_{1}$. Consequently, a right-directional acceleration will lead to a left-directed fictitious force and a subsequent displacement of the $S 3$ layer toward the PC. This positional variation decreases the width of the $A 1$ air spacer and produces a blueshift in the cavity frequency $\omega_{0} \rightarrow \omega_{0}+\epsilon(\alpha)$. An opposite displacement will produce a redshift to the cavity frequency $\omega_{0} \rightarrow \omega_{0}-\epsilon(\alpha)$ (we assume $\epsilon>0$ ). This process is modeled by the following modified effective Hamiltonian:

$$
H_{\epsilon}=\left(\begin{array}{cc}
\omega_{0}-i \gamma & \gamma \\
\gamma & \omega_{0}+\epsilon+i \gamma
\end{array}\right)
$$

where we have assumed that the EP condition $\kappa=\gamma$ is satisfied and $\epsilon=\epsilon(\alpha)=K \chi \alpha$. The new set of perturbed eigenfrequencies are

$$
\omega_{ \pm}=\omega_{\mathrm{EP}}+\frac{\epsilon}{2} \pm \frac{\sqrt{\epsilon^{2}+4 i \gamma \epsilon}}{2} .
$$

For small frequency detuning $\epsilon \ll \gamma$ we have

$$
\begin{aligned}
& \omega_{+} \approx \omega_{\mathrm{EP}}+\frac{\sqrt{2}}{2}(1+i) \sqrt{\epsilon}, \\
& \omega_{-} \approx \omega_{\mathrm{EP}}-\frac{\sqrt{2}}{2}(1+i) \sqrt{\epsilon},
\end{aligned}
$$

while for large detuning $\epsilon \gg \gamma$ we recover a standard linear shift, i.e.,

$$
\begin{aligned}
& \omega_{+} \approx \omega_{\mathrm{EP}}+\epsilon+i \gamma, \\
& \omega_{-} \approx \omega_{\mathrm{EP}}-i \gamma .
\end{aligned}
$$

Our interest, of course, will be focused on the parametric domain where the conditions (i.e., $\epsilon \ll \gamma$ ) for the validity of Eq. (9) are satisfied. In fact, this enhanced response of the eigenfrequency shifts carries over to the scattering domain where it surfaces as a shift of the poles $\omega^{S}$ of the scattering

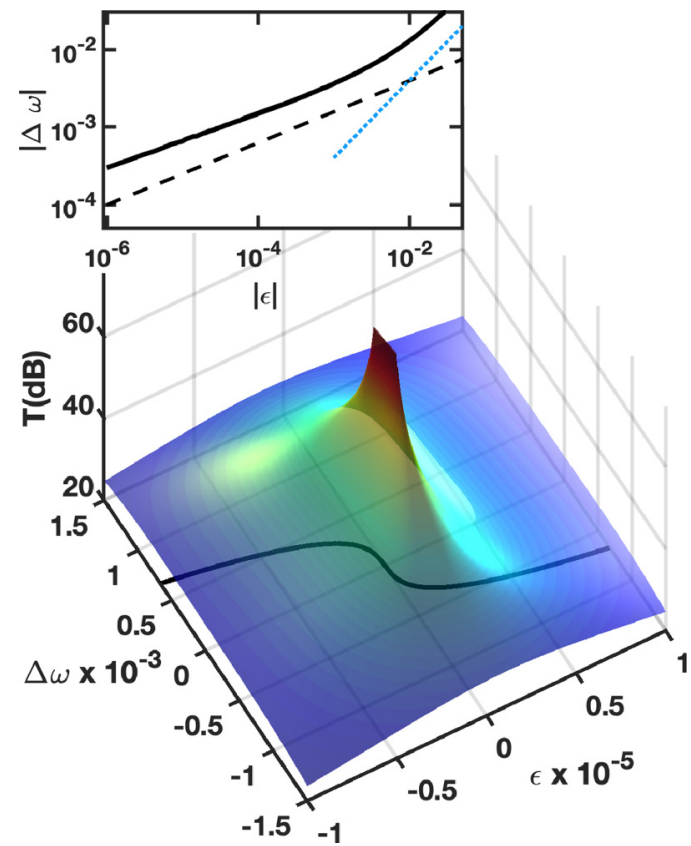

FIG. 5. Theoretical value of transmittance $T$ (in $\mathrm{dBs}$ ) vs frequency detuning $\Delta \omega=\omega_{\mathrm{EP}}-\omega$ from the EP and resonant perturbation $\epsilon$. In calculations, $\kappa=\gamma=0.3, w_{e}=10^{-4}$. The solid line indicates the resonance detuning (at peak transmittance) for each $\epsilon$-value. In the inset we plot on a double logarithmic plot $\left|\Delta \omega\left(T_{\text {peak }}\right)\right|$ versus a perturbation $|\epsilon|$. For small perturbations we find a square root-like behavior as expected from Eq. (9) (Best fit is shown with the black dashed line having slope $\approx 0.4)$. For larger perturbations we find a transition to a linear behavior $\left|\Delta \omega\left(T_{\text {peak }}\right)\right| \propto|\epsilon|$ (indicated by blue dotted line) as expected from Eq. (10).

matrix $S$. The associated $2 \times 2$ scattering matrix takes the form [46]

$$
S=-I-i W^{T} \frac{1}{H_{\epsilon}-\omega I-\frac{i}{2} W W^{T}} W, \quad W_{n m}=\sqrt{2 w_{e}} \delta_{n m},
$$

where $I$ is the $2 \times 2$ identity matrix and $W$ is the coupling matrix that connects the two modes to the free space with a coupling strength $w_{e}$. The poles of the scattering matrix are found easily from the secular equation $\operatorname{det}\left[H_{\epsilon}-\frac{i}{2} W W^{T}-\omega I\right]=0$. We get

$$
\omega_{ \pm}^{S}=\omega_{\mathrm{EP}}+\frac{\epsilon-2 i w_{e}}{2} \pm \frac{\sqrt{\epsilon^{2}+4 i \gamma \epsilon}}{2},
$$

which in the limit of $w_{e} \rightarrow 0$ collapses to the eigenfrequencies of the dimer systems Eq. (8). In fact, both $\omega_{ \pm}$in Eq. (8) and the poles $\omega_{ \pm}^{S}$ in Eq. (12) demonstrate the same qualitative features on their dependence on $\epsilon$. Let us analyze these features by referring, for simplicity, to the poles - the latter being directly relevant to the proposed acceleration measurement scheme. For small $\epsilon \ll \gamma$ both poles are shifted away from $\omega_{\mathrm{EP}}$ in opposite directions with a rate that is proportional to $\sqrt{\gamma \epsilon}$. At the same time, $\operatorname{Im}\left(\omega_{+}^{S}\right)$ becomes positive and larger, leading to a narrow, high- $Q$, transmittance peak. In contrast, the $\operatorname{Im}\left(\omega_{-}^{S}\right)$ broadens with the same rate $\sqrt{\gamma \epsilon}$ leading to a low- $Q$ resonant transmission peak (see Fig. 5). In fact, the linewidth broadening of the $\omega_{-}^{S}$ pole occurs at the same rate as a shift of the resonant frequencies $\omega_{+}^{S}$. As a consequence, these resonant 
modes have indistinguishable peaks in the transmission spectrum $T(\omega)$. Once the detuning becomes large enough, $\epsilon \gg \gamma$, the $\omega_{-}^{S}$ pole moves back to $\omega_{\mathrm{EP}}$ and its position remains unchanged with a further increase of $\epsilon$. Its linewidth $\operatorname{Im}\left(\omega_{-}^{S}\right)$ continues to broaden (due to negative imaginary component $-i \gamma$ ) leading to a suppression of the transmittance (see Fig. 5). In contrast, the other pole $\omega_{+}^{S}$ is shifted further away from the $\omega_{\mathrm{EP}}$ with a rate that is proportional to $\epsilon$ and at the same time it becomes narrower (due to positive imaginary component $+i \gamma)$. These features are nicely reflected in the transmission spectrum of our structure; see Fig. 5. The latter is analytically evaluated from Eq. (11):

$$
\begin{aligned}
T(\omega) & \equiv\left|S_{12}(\omega)\right|^{2} \\
& =\left|\frac{2 i \kappa w_{e}}{\left[\Delta \omega-i\left(\gamma+w_{e}\right)\right]\left[\Delta \omega+\epsilon+i\left(\gamma-w_{e}\right)\right]-\kappa^{2}}\right|^{2},
\end{aligned}
$$

where $\Delta \omega=\omega_{\mathrm{EP}}-\omega$ while at EP. $\kappa=\gamma$ and resonant frequencies are given by Eq. (12). Also, we point out that the rapid suppression of one of the transmission peaks (associated with $\omega_{-}^{S}$ ) and the simultaneous formation of a high- $Q$ resonant mode at $\omega_{+}^{S}$ results in a clearly resolved transmittance peaks in the spectrum without the concern of resolution digression due to (partially) overlapping resonances. As opposed to recent proposals that utilize EP for the realization of ring-laser gyroscopes, our design is operating below the lasing threshold and therefore must not be affected by quantum-noise effects [47-49]. In contrast to the ring-laser resonators, the Fabry-Pérot multilayer supports only one high- $Q$ defect mode within the band gap (the other mode is overdamped and suppressed). This feature leads to an improved spectral resolution of the device and thus to the precision of the measurement. Another important advantage of our design is its tolerance to global temperature variations of the multilayer: In particular, a temperature variation that induces a change in the thickness or the refractive index of the silicon layers will affect uniformly the whole multilayer, producing the same shift in both resonant frequencies $\Delta \omega_{+}=\Delta \omega_{-}$. Consequently, such thermally induced variations will not shift the system from the EP-as opposed to perturbations that affect only one of the modes: $\Delta \omega_{+}=0, \Delta \omega_{-} \neq 0$. Such behavior provides an additional degree of robustness of the device against parasitic (thermal) perturbations.

Moreover, as was recently shown both numerically [24,27] and experimentally [26,50], in $\mathcal{P} T$-symmetric detectors based on whispering-gallery mode (ring) resonators the motion of a particle or a cantilever in close proximity to the resonators introduces an evanescent coupling between clockwise $(\mathrm{CW})$ and counterclockwise (CCW) modes of such ring resonators, or in the case of coupled nanobeam cavities, it introduces coupling between the modes of the two cavities [29]. In all these cases, we come up with a perturbation of the off-diagonal elements of the non-Hermitian Hamiltonian, which lifts the EP degeneracy. In the case in which the motion of the cantilever or the particle is toward the ring resonator, the coupling between CW-CWW modes is increased compared to that at the EP, i.e., the system undergoes a transition to the so-called exact phase regime where the eigenfrequencies are purely real and can be easily measured via the transmittance or the reflectance spec- tra. On the other hand, an opposite motion of the cantilever or of the particle (i.e., away from the resonator) leads the system to the so-called broken phase regime, where there is no resonance splitting (only the imaginary parts of the resonances deviate from one another). This implies that such sensors can only measure the proximity of the cantilever/particles in one direction. Therefore, such measurement protocols are incompatible with avionic sensing, where sensing directional motion is critical. In contrast, the accelerometer design that we have proposed here relies on the perturbation of the diagonal elements of the effective Hamiltonian, thus providing the possibility to sense the direction of the acceleration.

The above accelerometer proposal can be further extended to include $N$ Fabry-Pérot cavities. In this case, the $2 \times 2$ Hamiltonian from Eqs. (5) and (7) will be extended to an $(N \times N)$-dimensional matrix. It was shown that such highdimensional systems, under an appropriate design of the coupling elements, can support the coalescence of more modes, leading to a higher-order EP degeneracy. In such cases, a small perturbation can lead to a degeneracy lifting that is proportional to an $n$ th-order root $(2<n \leqslant N)$ : $\omega_{ \pm}-\omega_{\mathrm{EP}} \propto$ $\sqrt[n]{\epsilon},(\epsilon \ll 1)$, thus enhancing the sensitivity level of the sensor [25]. Inverse design techniques, which might also incorporate signal-to-noise constraints, might be a useful tool in designing such hypersensitive avionic sensors.

\section{CONCLUSION}

In conclusion, we proposed a conceptual design of an optomechanical accelerometer based on two coupled FabryPérot cavities with a balanced amount of gain and loss. The structure is tuned to be at an EP in the absence of any acceleration. One of the layers is supposed to be attached to spring mass in order to sense the in-plane acceleration. The applied acceleration results in a displacement of the silicon layer with respect to the rest of the stack. This displacement is proportional to the acceleration and triggers a resonant frequency detuning of the high- $Q$ mode, and thus removal of the EP degeneracy. Using a theoretical model based on coupled mode theory, we have described the effects of the acceleration in the two defect modes that form the EP. We found that the resonant peak of the transmittance shifts in a square-root manner as a response to the displacement (and thus acceleration) of the test mass. Detailed simulations confirmed this enhanced sensitivity. Our proposed scheme is superior to conventional acceleration sensors for which the resonant transmission peak shifts proportionally to the test-mass displacement (and thus to the acceleration).

\section{ACKNOWLEDGMENTS}

This research was supported by the Office of Naval Research via Grant No. N00014-19-1-2480 and by the Air Force Office of Scientific Research via Grant No. FA9550-10-10433

\section{APPENDIX A: RESPONSE FUNCTION}

Consider a damped mechanical harmonic oscillator with mass $m$, spring constant $k$, natural frequency $\omega_{n} \equiv \sqrt{\frac{k}{m}}$, attenuation parameter $\gamma$, and applied external force $F_{\text {appl }}$. Such a 
system is described by the following differential equation of motion:

$$
m \ddot{x}+m \gamma \dot{x}+m \omega_{n}^{2} x=F_{\text {appl }},
$$

which after Fourier transform is written as

$$
-\omega x(\omega)+i \gamma x(\omega)+\omega_{n}^{2} x(\omega)=\frac{F_{\text {appl }}(\omega)}{m} .
$$

Substituting $\alpha(\omega)=F_{\text {appl }}(\omega) / m$, one finally has

$$
x(\omega)=\chi(\omega) \alpha(\omega),
$$

where

$$
\chi(\omega)=\frac{1}{\omega_{n}^{2}-\omega^{2}+i \frac{\omega_{n} \omega}{Q}}
$$

is the susceptibility of the mechanical oscillator, which defines the response of the oscillator to external frequency-dependent excitations associated with a force $F_{\text {appl }}(\omega)$. Above, $Q=$ $\omega_{n} / \gamma$ is the quality factor of the oscillator. From Eq. (A4) it is seen that in the low-frequency limit $\left(\omega \ll \omega_{n}\right)$ the susceptibility is $\chi\left(\omega \ll \omega_{n}\right) \propto \frac{1}{\omega_{n}^{2}}$, corresponding to a flat frequency response of the mechanical oscillator. At the resonance frequency $\omega_{n}$ the response of the oscillator is enhanced by the $Q$ factor: $\chi\left(\omega_{n}\right)=-i \frac{Q}{\omega_{n}^{2}}$. At the same time, at frequencies much higher than the natural frequency, the susceptibility decays in a square manner as a function of frequency: $\chi\left(\omega \gg \omega_{n}\right) \propto \frac{1}{\omega^{2}}$, leading to a very weak response of the mechanical system to excitations with frequencies higher than the resonant frequency. Such functional behavior leads to the conclusion that an accelerometer, in order to be able to measure accelerations with different frequencies with the same efficiency (i.e., to be a broadband sensor), has to operate well below the natural frequency $\omega_{n}$ where susceptibility $\chi$ provides a flat frequency response. In this case, on the other hand, the natural frequency defines the sensitivity of the accelerometer, leading to a fundamental tradeoff between the sensitivity of the mechanical accelerometer sensitivity and its bandwidth.

\section{APPENDIX B: THERMAL NOISE}

Any mechanical oscillator is subject to parasitic random oscillations due to a Brownian motion within the surrounding media. This random excitation due to thermal noise is translated into a force $F_{\text {noise }}=m \alpha_{\text {noise }}$, which sets the fundamental resolution limit of an accelerometer. The mechanical system, which is subject to random excitations due to Brownian motion, will follow the differential equation

$$
i m v(\omega)+m \gamma v(\omega)-i \frac{k}{\omega} v(\omega)=F_{\text {noise }}(\omega),
$$

which can be obtained from Eq. (A1) by substituting $v \equiv \dot{x}$ and $F_{\text {noise }}=F_{\text {appl }}$. Substituting the expressions $Q=\omega_{0} / \gamma$ and $f=\omega /(2 \pi)$ in the above equation leads to the following expression for the velocity:

$$
\nu(f)=\frac{F_{\text {noise }}(f)}{\gamma m\left[1+i Q\left(\frac{f}{f_{n}}-\frac{f_{n}}{f}\right)\right]} .
$$

The average (over spectrum) force due to thermal oscillations is described by the root mean square (RMS) value $\left\langle F_{\text {noise }}\right\rangle$, where the overbar indicates a spectral averaging. We have

$$
\left\langle F_{\text {noise }}\right\rangle^{2}=\int_{f_{1}}^{f_{1}+\Delta f} \frac{F_{\text {noise }}^{2}(f)}{\Delta f} d f=\int_{f_{1}}^{f_{1}+\Delta f} \overline{F_{\text {noise }}^{2}(f)} d f .
$$

Assuming that the thermal noise is white noise, we can substitute the force spectral density with a constant, i.e., $\overline{F_{\text {noise }}^{2}(f)}=$ const. Therefore, Eq. (B3) can be further simplified to

$$
\left\langle F_{\text {noise }}\right\rangle^{2}=\overline{F_{\text {noise }}} \Delta f,
$$

where $\Delta f$ is the bandwidth of the measured accelerations, i.e., the inverse of time averaging of the processed signal, meaning that the noise signal with frequencies above $f_{1}+\Delta f$ and below $f_{1}$ will be averaged to zero, and thus will not affect the measurements. Then, from Eq. (B2) one obtains

$$
\begin{aligned}
\overline{\nu^{2}} & =\frac{\overline{F_{\text {noise }}^{2}}}{\left|\gamma m\left[1+i Q\left(\frac{f}{f_{n}}-\frac{f_{n}}{f}\right)\right]\right|^{2}} \\
& =\frac{\overline{F_{\text {noise }}^{2}}}{\gamma^{2} m^{2}\left[1+Q^{2}\left(\frac{f}{f_{n}}-\frac{f_{n}}{f}\right)^{2}\right]} .
\end{aligned}
$$

The mean kinetic energy stored in the mechanical system is defined as $E=\frac{1}{2} m\langle v\rangle^{2}$, where $\langle v\rangle$ is the RMS velocity of the oscillator due to thermal noise. On the other hand, the total kinetic energy of the thermal noise oscillations from all the frequencies $(f \in[0, \infty])$ is $E=\frac{1}{2} k_{b} T$, where $k_{b}$ is the Boltzmann constant and $T$ is the temperature. The equipartition theorem leads to

$$
E=\frac{1}{2} k_{b} T=\frac{1}{2} m\langle v\rangle^{2}=\frac{1}{2} m \int_{0}^{\infty} \overline{v^{2}} d f .
$$

Substituting Eq. (B5) into Eq. (B6), one can write

$$
\frac{1}{2} k_{b} T=\frac{\overline{F_{\text {noise }}}}{4 \pi \gamma m} \int_{0}^{\infty} \frac{Q d\left(\frac{f}{f_{n}}\right)}{\left(1+Q^{2}\left(\frac{f}{f_{n}}-\frac{f_{n}}{f}\right)^{2}\right)} .
$$

We can make further progress with the integration of Eq. (B7) by substituting $\frac{f}{f_{n}}=e^{x}$. We get

$$
\frac{1}{2} k_{b} T=\frac{\overline{F_{\text {noise }}^{2}}}{8 \gamma m},
$$

leading to

$$
\overline{F_{\text {noise }}^{2}}=4 k_{b} T \gamma m \text {. }
$$

Thus, the spectral density of the thermal noise equivalent acceleration $\alpha_{\mathrm{th}}=\sqrt{\frac{F_{\text {nise }}^{2}}{m^{2}}}$ can be written as

$$
\alpha_{\mathrm{th}}=\sqrt{\frac{4 k_{b} T \gamma}{m}}=\sqrt{\frac{4 k_{b} T \omega_{n}}{m Q}} .
$$

Then from Eqs. (B4) and (B5), the RMS acceleration due to the thermal noise is

$$
\left\langle\alpha_{\text {noise }}\right\rangle=\sqrt{\frac{\left\langle F_{\text {noise }}\right\rangle^{2}}{m^{2}}}=\sqrt{\frac{\overline{F_{\text {noise }}^{2}} \Delta f}{m^{2}}}=\alpha_{\text {th }} \sqrt{\Delta f},
$$

which is the lower bound of the measured accelerations within the frequency range $\Delta f$. At the same time from Eq. (A3), 
$\overline{x_{\text {noise }}^{2}}=|\chi(\omega)|^{2} \frac{\overline{F_{\text {noise }}^{2}}}{m^{2}}$, and thus one can obtain the spectral density of the noise displacement,

$$
\begin{aligned}
x_{\mathrm{th}}(\omega) & =\sqrt{\overline{x_{\text {noise }}^{2}}}=|\chi(\omega)| \alpha_{\mathrm{th}} \\
& =\left(\frac{4 k_{b} T \omega_{n}}{m Q\left(\left(\omega_{n}^{2}-\omega^{2}\right)^{2}+\left(\frac{\omega_{n} \omega}{Q}\right)^{2}\right)}\right)^{\frac{1}{2}},
\end{aligned}
$$

which at the low-frequency limit $\left(\omega \ll \omega_{n}\right)$, where $\chi(\omega)=$ $\frac{1}{\omega_{n}^{2}}$, translates to

$$
x_{\text {th }}\left(\omega \ll \omega_{n}\right)=\frac{\alpha_{\text {th }}}{\omega_{n}^{2}}=\sqrt{\frac{4 k_{b} T}{m Q \omega_{n}^{3}}}=\text { const, }
$$

indicating flat response to the thermal noise within the lowfrequency limit.
[1] G. Krishnan, C. U. Kshirsagar, G. K. Ananthasuresh, and N. Bhat, Micromachined high-resolution accelerometers, J. Indian Inst. Sci. 87, 333 (2007).

[2] Y. Shindo, T. Yoshikawa, and H. Mikada, A large scale seismic sensing array on the seafloor with fiber optic accelerometers, Proc. IEEE Sens. 1, 1767 (2002).

[3] P. S. de Brito Andre and H. Varum, Accelerometers: Principles, Structure and Applications (Nova Science, Hauppauge, NY, 2013).

[4] M. Bao, Micro Mechanical Transducers: Pressure Sensors, Accelerometers and Gyroscopes (Elsevier, Amsterdam, 2000).

[5] C. Acar and A. M. Shkel, Experimental evaluation and comparative analysis of commercial variable-capacitance MEMS accelerometers, J. Micromech. Microeng. 13, 634 (2003).

[6] S. Tadigadapa and K. Mateti, Piezoelectric MEMS sensors: state-of-the-art and perspectives, Meas. Sci. Technol. 20, 092001 (2009).

[7] C. Liu, A. M. Barzilai, J. K. Reynolds, A. Partridge, T. W. Kenny, J. D. Grade, and H. K. Rockstad, Characterization of a high-sensitivity micromachined tunneling accelerometer with micro-g resolution, J. Microelectromech. Syst. 7, 235 (1998).

[8] U. Krishnamoorthya, R. H. Olsson IIIa, G. R. Bogartb, M. S. Bakera, D. W. Carrb, T. P. Swilera, and P. J. Clewsa, Inplane MEMS-based nano-g accelerometer with sub-wavelength optical resonant sensor, Sens. Actuator. A 145-146, 283 (2008).

[9] K. Zandi, B. Wong, J. Zou, R. V. Kruzelecky, W. Jamroz, and Y. Peter, In-plane silicon-on-insulator optical MEMS accelerometer using waveguide fabry-perot microcavity with silicon/air brag mirror, in 2010 IEEE 23rd International Conference on Micro Electro Mechanical Systems (MEMS) (IEEE, Piscataway, NJ, 2010), p. 839

[10] W. Noell, Applications of SOI-based optical MEMS, IEEE J. Sel. Top. Quantum Electron. 8, 148 (2002).

[11] T. A. Berkoff and A. D. Kersey, Experimental demonstration of a fiber bragg grating accelerometer, IEEE Photon. Technol. Lett. 8, 1677 (1996).

[12] A. G. Krause, M. Winger, T. D. Blasius, Q. Lin, and O. Painter, A microchip optomechanical accelerometer, Nat. Photon. 6, 768 (2012).

[13] Y. L. Li and P. F. Barker, Characterization and testing of a micro-g whispering gallery mode optomechanical accelerometer, J. Light. Technol. 36, 3919 (2018).

[14] A. Schliesser, O. Arcizet, G. Riviere, G. Anetsberger, and T. J. Keppenberg, Resolved-sideband cooling and position measurements of a micromechanical oscillator close to the Heisenberg uncertainty limit, Nat. Phys. 5, 509 (2009).

[15] C. A. Regal, J. D. Teufel, and K. W. Lehnert, Measuring nanomechanical motion with a microwave cavity interferometer, Nat. Phys. 4, 555 (2008).
[16] V. B. Braginsky, Measurement of Weak Forces in Physics Experiments (University of Chicago Press, Chicago, 1977)

[17] M. Wu, A. C. Hryciw, C. Healey, D. P. Lake, H. Jayakumar, M. R. Freeman, J. P. Davis, and P. E. Barclay, Dissipative and Dispersive Optomechanics in a Nanocavity Torque Sensor, Phys. Rev. X 4, 021052 (2014).

[18] B-B. Li, J. Bilek, B. H. Ulrich, L. S. Madsen, S. Forstner, V. Prakash, C. Schäfermeier, T. Gehring, W. P. Bowen, and U. L. Andersen, Quantum enhanced optomechanical magnetometry, Optica 5, 850 (2018).

[19] M. A. Taylor, J. Janousek, V. Daria, J. Knittel, B. Hage, H.-A. Bachor, and W. P. Bowen, Biological measurement beyond the quantum limit, Nat. Photon. 7, 229 (2013).

[20] C. E. Rüter, K. G. Makris, R. El-Ganainy, D. N. Christodoulides, M. Segev, and D. Kip, Observation of parity-time symmetry in optics, Nat. Phys. 6, 192 (2010).

[21] Ş. K. Özdemir, S. Rotter, F. Nori, and L. Yang, Parity-time symmetry and exceptional points in photonics, Nat. Mater. 18, 783 (2019).

[22] J. Schindler, A. Li, M. C. Zheng, F. M. Ellis, and T. Kottos, Experimental study of active LRC circuits with PT symmetries, Phys. Rev. A 84, 040101(R) (2011).

[23] X. Zhu, H. Ramezani, C. Shi, J. Zhu, and X. Zhang, PTSymmetric Acoustics, Phys. Rev. X 4, 031042 (2014).

[24] J. Wiersig, Enhancing the Sensitivity of Frequency and Energy Splitting Detection by using Exceptional Points: Applications to Microcavity Sensors for Single Particle Detection, Phys. Rev. Lett. 112, 203901 (2014).

[25] H. Hodaei, A. U. Hassan, S. Wittek, H. Garcia-Garcia, R. ElGanainy, D. Christodoulides, and M. Khajavikhan, Enhanced sensitivity at higher-order exceptional points, Nature (London) 548, 187 (2017).

[26] W. Chen, Ş. K. Özdemir, G. Zhao, J. Wiersig, and L. Yang, Exceptional points enhance sensing in an optical microcavity, Nature (London) 548, 192 (2017).

[27] Q. Zhong, J. Ren, M. Khajavikhan, D. N. Christodoulides, Ş. K. Özdemir, and R. El-Ganainy, Sensing with Exceptional Surfaces in Order to Combine Sensitivity with Robustness, Phys. Rev. Lett. 122, 153902 (2019).

[28] Z. P. Liu, J. Zhang, Ş. K. Özdemir, B. Peng, H. Jing, X. Y. Lü, C. W. Li, L. Yang, F. Nori, and Y. X. Liu, Metrology with $\mathcal{P} T$ Symmetric Cavities: Enhanced Sensitivity Near the $\mathcal{P} T$-Phase Transition, Phys. Rev. Lett. 117, 110802 (2016)

[29] S. Zhang, Z. Yong, Y. Zhang, and S. He, Parity-time symmetry breaking in coupled nanobeam cavities, Sci. Rep. 6, 24487 (2016).

[30] P.-Y. Chen and J. Jung, PT Symmetry and Singularity-Enhanced Sensing Based on Photoexcited Graphene Metasurfaces, Phys. Rev. Appl. 5, 064018 (2016). 
[31] M. P. Hokmabadi, A. Schumer, D. N. Christodoulides, and M. Khajavikhan, Non-Hermitian ring laser gyroscopes with enhanced Sagnac sensitivity, Nature (London) 576, 70 (2019).

[32] Y. H. Lai, Y. K. Lu, M. G. Suh, Z. Yuan, and K. Vahala, Observation of the exceptional-point-enhanced Sagnac effect, Nature (London) 576, 65 (2019).

[33] B. Saadany, M. Malak, M. Kubota, F. Marty, Y. Mita, D. Khalil, and T. Bourouina, Free-space tunable and drop optical filters using vertical Bragg mirrors on silicon, IEEE J. Sel. Top. Quantum Electron. 12, 1480 (2006).

[34] M. W. Pruessner, T. H. Stievater, and W. S. Rabinovich, Integrated waveguide Fabry-Perot microcavities with silicon/air Bragg mirrors, Opt. Lett. 32, 533 (2007).

[35] A. Polman, Erbium implanted thin film photonic materials, J. Appl. Phys. 82, 1 (1997).

[36] L. Yang, T. Carmon, B. Min, S. M. Spillane, and K. J. Vahala, Erbium-doped and Raman microlasers on a silicon chip fabricated by the sol-gel process, Appl. Phys. Lett. 86, 091114 (2005).

[37] B. Peng, S. K. Ozdemir, F. Lei, F. Monifi, M. Gianfreda, G. L. Long, S. Fan, F. Nori, C. M. Bender, and L. Yang, Parity-timesymmetric whispering-gallery microcavities, Nat. Phys. 10, 394 (2014).

[38] H. H. Li, Refractive index of silicon and germanium and its wavelength and temperature derivatives, J. Phys. Chem. Ref. Data 9, 561 (1993).

[39] COMSOL Multiphysics v. 5.5., COMSOL AB, Stockholm, Sweden, www.COMSOL.com (2020).

[40] B. C. Stipe, H. J. Mamin, T. D. Stowe, T. W. Kenny, and D. Rugar, Noncontact Friction and Force Fluctuations Between Closely Spaced Bodies, Phys. Rev. Lett. 87, 096801 (2001).
[41] K. Y. Yasumura, T. D. Stowe, E. M. Chow, T. Pfafman, T. W. Kenny, B. C. Stipe, and D. Rugar, Quality factors in micronand submicron-thick cantilevers, J. Microelectromech. Syst. 9, 117 (2000).

[42] A. N. Cleland and M. L. Roukes, Noise processes in nanomechanical resonators, J. Appl. Phys. 92, 2758 (2002).

[43] J. D. Joannopoulos, S. G. Johnson, R. D. Winn, and R. D. Weade, Photonic Crystals: Molding the Flow of Light (Princeton University Press, Princeton, NJ, 2008).

[44] P. Markos and C. M. Soukoulis, Wave Propagation: From Electrons to Photonic Crystals and Left-Handed Materials (Princeton University Press, Princeton, NJ, 2008).

[45] H. A. Macleod, Thin-film Optical Filters (CRC, Boca Raton, FL, 2017).

[46] U. Kuhl, F. Mortessagne, E. Makri, I. Vitebskiy, and T. Kottos, Waveguide photonic limiters based on topologically protected resonant modes, Phys. Rev. B 95, 121409(R) (2017).

[47] W. Langbein, No exceptional precision of exceptional-point sensors, Phys. Rev. A 98, 023805 (2018).

[48] A. E. Siegman, Excess spontaneous emission in non-Hermitian optical systems. II. Laser oscillators, Phys. Rev. A 39, 1253 (1989).

[49] N. A. Mortensen, P. A. D. Gonçalves, M. Khajavikhan, D. N. Christodoulides, C. Tserkezis, and C. Wolff, Fluctuations and noise-limited sensing near the exceptional point of parity-timesymmetric resonator systems, Optica 5, 1342 (2018).

[50] C. Wang, X. Jiang, G. Zhao, M. Zhang, C. W. Hsu, B. Peng, A. D. Stone, L. Jiang, and L. Yang, Electromagnetically induced transparency at a chiral exceptional point, Nat. Phys. 16, 334 (2020). 